CLINICAL STUDY

\title{
Endogenous sex hormones and the prospective association with cardiovascular disease and mortality in men: the Tromso Study
}

\author{
Torkel Vikan $^{1,4}$, Henrik Schirmer ${ }^{2,3}$, Inger Njølstad ${ }^{3}$ and Johan Svartberg ${ }^{1,4}$ \\ ${ }^{1}$ Division of Internal Medicine and ${ }^{2}$ Department of Cardiology, University Hospital of North Norway, Tromsø 9038, Norway, ${ }^{3}$ Institute of Community \\ Medicine and ${ }^{4}$ Institute of Clinical Medicine, University of Tromsø, Tromsø 9037, Norway
}

(Correspondence should be addressed to T Vikan at Division of Internal Medicine, University Hospital of North Norway; Email: torkel.vikan@unn.no)

\begin{abstract}
Objective: To study the impact of endogenous testosterone levels in community-dwelling men on later risk for myocardial infarction (MI) and all-cause, cardiovascular disease (CVD), and ischemic heart disease (IHD) mortality.

Design: Population-based prospective cohort study.

Methods: For the analyses, we used a cohort of 1568 randomly selected men, with sex-hormone data participating in the fourth Tromsø Study (1994-1995). Defined end points were first-ever MI (fatal or nonfatal), all-cause, CVD, and IHD mortality. A committee performed thorough ascertainment of end points, following a detailed protocol. Complete ascertainment of end points was until 30 September 2007 for all-cause mortality, until 31 December 2005 for CVD/IHD mortality, and until 31 December 2004 for first-ever MI. The prospective association between total and free testosterone and end points were examined using Cox proportional hazard regression, allowing for multivariate adjustment for age and cardiovascular risk factors.

Results: During follow-up, there were 395 deaths from all causes, 130 deaths from CVD and 80 deaths from IHD, while 144 men experienced a first-ever MI. There was a significant increase in all-cause mortality risk for men with free testosterone in the lowest quartile $(<158 \mathrm{pmol} / \mathrm{l})$ compared with the higher quartiles after age adjustment hazard ratios (HR 1.24, 95\% confidence interval, CI 1.01-1.53) and after multivariate adjustments (HR 1.24, 95\% CI 1.01-1.54). Total testosterone was not associated with mortality risk. Likewise, there were no significant changes in risk for first-ever MI across different total or free testosterone levels.

Conclusion: Men with free testosterone levels in the lowest quartile had a $24 \%$ increased risk of all-cause mortality.
\end{abstract}

European Journal of Endocrinology 161 435-442

\section{Introduction}

Men experience a gradual decrease in testosterone levels from the age of 30 and onward (1). Male ageing and male hypogonadism share many common traits, and the use of testosterone supplementation for male well being, as a potential fountain of youth, is increasing (2). This is in spite of limited knowledge on benefits and long-term safety, as a large randomized controlled trial (RCT) on long-term effects of testosterone supplementation has never been conducted. The primary concerns with supplementation, namely prostate cancer and cardiovascular disease (CVD; although the latter was more a concern under the estrogen-protection orthodoxy), have not been justified by epidemiological studies. On the contrary, higher physiological testosterone levels are now receiving attention as a beneficial modulator of known cardiovascular risk factors (3). However, in prospective population-based studies, low testosterone levels have not been associated with an increased risk of coronary events (4-9), and for a long time, observational studies failed to reveal significant associations between low testosterone levels and mortality. Within the last year, two reports from large prospective studies were published demonstrating that men with lower testosterone levels had a higher risk of death from all-cause and CVD mortality $(10,11)$. It was suggested that the lack of associations reported from earlier studies attributed to small study samples and low accuracy in the measurement of sex hormones.

Our aims were to study the impact of endogenous sex hormone levels in men on later risk for myocardial infarction (MI) and death from all-cause, CVD, and ischemic heart disease (IHD), in a cohort from the fourth Tromsø Study. We hypothesized that lower testosterone levels in community-dwelling men would be associated with a higher risk for MI, and an increased risk for death. 


\section{Materials and methods}

\section{Study population}

The Tromsø Study is a population-based prospective study with repeated health surveys, primarily focusing on cardiovascular and other chronic diseases. The population of the fourth Tromsø Study (1994-1995) is described in detail previously (12). From the fourth Tromsø Study, data on sex hormones were available on a randomly selected subsample of 1579 men. Eleven men had serum testosterone levels $<0.5 \mathrm{nmol} / \mathrm{l}$, indicating biochemical or surgical castration and were excluded, leaving 1568 men to be used for analysis of all-cause, CVD, and IHD mortality. For analysis of incident MI, 1318 men were available after exclusion of 246 men with self-reported and/or known MI before the baseline examination and four men who had moved from the area.

\section{Definition and ascertainment of end points}

The outcomes were as follows: first-ever fatal or non-fatal MI (for simplicity first-ever MI in the following), all-cause, CVD, and IHD mortality.

Acute coronary events were categorized according to clinical presentation, electrocardiographic changes, levels of myocardial biomarkers, and, if applicable, results of coronary angiography, echocardiography, and/or autopsy. Assessment of the cardiovascular morbidity end points was performed by an end-point committee based on medical records from the University Hospital of North Norway, the only hospital serving the Tromsø population, and event ascertainment followed a detailed protocol. The Norwegian Registry of Vital Statistics provided information on death and emigration. Causes of deaths were obtained from the Causes of Death Registry at Statistics Norway. All possible events, including possible out-of-hospital fatal MIs, strokes, and sudden deaths, were reviewed according to the event protocol by examining medical records, death certificates, and autopsy records.

Data on all-cause mortality were obtained from The Norwegian Registry of Vital Statistics, while data on CVD (ICD-9: 390-458, 250, 795, 798 and ICD-10: I00-I99, R96, R98, R99) and IHD (ICD-9: 410-414 and ICD-10: I20-I25) mortality were obtained from the Causes of Death Registry at Statistics Norway. Complete assessment of end points was until 31 December 2004 for first-ever MI, until 31 December 2005 for mortality from CVD and IHD, and until 30 September 2007 for all-cause mortality. For assessment of incidence of firstever MI, the individual was censored on the registered date of migration, emigration, or death. For assessment of all-cause mortality, mortality from CVD, and mortality from IHD, the individual was censored on the registered date of emigration (migration status not necessary for end-point ascertainment of mortality since this was based on a national registry).

\section{Baseline characteristics}

Height and weight were measured in standing subjects wearing light clothing without shoes. Waist circumference was measured at the umbilical line according to written protocol. Body mass index (BMI; $\mathrm{kg} / \mathrm{m}^{2}$ ) was calculated. Blood pressure was recorded with an automatic device (Dinamap Vital Signs Monitor, Critikon Inc., Tampa, FL, USA) by specially trained personnel. Non-fasting blood samples were drawn between 0800 and $1600 \mathrm{~h}$. Serum total cholesterol and triglycerides were analyzed by enzymatic colorimetric methods with commercial kits (CHOD-PAP for cholesterol and GPO-PAP for triglycerides; BoehringerMannheim). Serum high-density lipoprotein (HDL) cholesterol was measured after precipitation of lower density lipoprotein with heparin and manganese chloride.

Self-administered questionnaires that included information about smoking habits, physical activity, medical history, and use of anti-hypertensive and lipid-lowering drugs were completed and checked by trained nurses.

\section{Sex hormones}

Serum samples from 1994 were analyzed for sex hormones in the autumn of 2001. All samples were stored frozen at $-70{ }^{\circ} \mathrm{C}$ until they were first thawed in 2001. The determination of total testosterone, estradiol $\left(\mathrm{E}_{2}\right)$, and sex hormone-binding globulin (SHBG) was performed on Immulite 2000 (Diagnostic Product Corp., Los Angeles, CA, USA). The intraand interassay coefficients of variation for the analyses were between 5 and 10\%. Free testosterone values were calculated from total testosterone and SHBG using a fixed albumin concentration according to Vermeulen et al. (13).

\section{Statistical analysis}

Normal distribution was assessed by skewness and histograms. $\mathrm{E}_{2}$ and triglycerides were not normally distributed, but assumed normal distribution after log transformation. When analyzed as continuous variables, the log value was used. Age-adjusted partial correlations were used for analysis of associations between baseline variables and sex hormones. The prospective associations between sex hormones and end points were examined using Cox proportional hazard regression. Sex hormones were modeled both as continuous variables with hazard ratios (HR) for 1 s.D. increase in hormone level, and as quartiles based on the entire population sample, with the lowest quartile used as the reference category. For all-cause mortality, total and free testosterone was also examined as dichotomous variables comparing the lowest quartile to the three higher. Free testosterone was also assessed 
as decentiles in order to identify a possible threshold effect for change in risk for all-cause mortality. For all regression analyses, two models were evaluated: one with adjustment only for age; and the other with multivariate adjustments including waist/hip ratio, $\mathrm{HDL} /$ cholesterol ratio, systolic blood pressure, current smoking, and self-reported diabetes. The ratios (waist/hip ratio and HDL/cholesterol ratio) were preferred over other measures of obesity and lipid status, as they are better predictors of MI (14), while vigorous physical activity did not associate with any of the end points in univariate models, and hence were not included as a potential confounder.

As testosterone could be a marker of prevalent cardiovascular (or other) disease, all analyses were repeated after exclusion of those who had an event during the first 2 years of follow-up, intending to account for potential subclinical prevalent disease at baseline that may have affected testosterone levels and then led to false prospective associations.

The proportional odds assumption was examined using log-minus-log plots, and the assumptions were met by all models. A $P$ value $<0.05$ was considered statistically significant, and all $P$ values presented are two tailed. The SPSS statistical software version 15.0 (SPSS Inc., Chicago, IL, USA) for windows was used for all analyses.

\section{Ethics}

The study protocol was approved by the Regional Committee for Medical and Health Research Ethics, North Norway, and informed consent was obtained from all the participants.

\section{Results}

\section{Testosterone and baseline characteristics}

The baseline characteristics are presented in Table 1. Age-adjusted partial correlations for total and free testosterone with covariates are also presented in Table 1. Both total and free testosterone was inversely correlated with age, but the correlation with free testosterone was much stronger than with total testosterone. Total testosterone was correlated with cardiovascular risk factors in a favorable manner for BMI, waist circumference, HDL-cholesterol, triglycerides, $\mathrm{HbA1c}$, systolic blood pressure, and diastolic blood pressure in an unfavorable manner for total cholesterol. The correlations with risk factors and free testosterone were generally weaker, but when significant, they were in the same direction as with total testosterone. Mean total and free testosterone levels were significantly higher in smokers and in men with a history of CHD. Diabetics had significantly lower total (but not free) testosterone. Testosterone levels did not differ by status of CVD or vigorous physical activity.

\section{Sex hormones and mortality}

During a mean average follow-up of 11.2 years (17 748 person years), 395 men died, corresponding to a mortality rate of 22.4 per 1000 person years. For CVD and IHD mortality, the average follow-up was 10.0 years (15 715 person years). There were 130 deaths from CVD and 80 deaths from IHD, corresponding to a CVD mortality rate of 8.5 per 1000 person years and an IHD mortality rate of 5.1 per 1000 person years respectively.

Table 1 Baseline characteristics of the 1568 participating men. Age-adjusted correlations with testosterone and free testosterone. The Tromsø Study 1994-1995.

\begin{tabular}{|c|c|c|c|}
\hline & Mean (S.D.) or (\%) & Total testosterone $\left(R^{\mathrm{a}}\right)$ & Free testosterone $\left(R^{\mathrm{a}}\right)$ \\
\hline Age (year) & $59.6(10.2)$ & $-0.09^{*}$ & $-0.42^{*}$ \\
\hline Waist/hip ratio & $0.92(0.06)$ & $-0.28^{*}$ & $-0.07^{\dagger}$ \\
\hline Total cholesterol $(\mathrm{mmol} / \mathrm{l})$ & $6.5(1.2)$ & $0.07^{\ddagger}$ & $0.11^{*}$ \\
\hline HDL-cholesterol (mmol//) & $1.4(0.4)$ & $0.10^{*}$ & -0.04 \\
\hline HDL/cholesterol ratio & $0.22(0.07)$ & $0.08^{\dagger}$ & $-0.09^{\dagger}$ \\
\hline Triglycerides $(\mathrm{mmol} / \mathrm{l})^{\mathrm{b}}$ & $1.7(1.0)$ & $-0.20^{\star}$ & 0.01 \\
\hline $\mathrm{HbA} 1 \mathrm{c}(\%)^{\mathrm{b}}$ & $5.5(0.7)$ & $-0.07^{\dagger}$ & -0.01 \\
\hline Testosterone $(\mathrm{nmol} / \mathrm{l})$ & $13.3(5.1)$ & - & $0.73^{*}$ \\
\hline SHBG $\left(\mathrm{nmol} / \mathrm{l}^{\mathrm{b}}\right.$ & $52.2(23.9)$ & $0.66^{*}$ & 0.05 \\
\hline Free testosterone $(\mathrm{pmol} / \mathrm{l})$ & $204(77)$ & $0.73^{*}$ & - \\
\hline Estradiol $(\mathrm{nmol} / \mathrm{l})^{\mathrm{b}}$ & $0.06(0.03)$ & $0.20^{*}$ & $0.15^{\star}$ \\
\hline Systolic blood pressure $(\mathrm{mmHg})$ & $141(20)$ & $-0.13^{\star}$ & $-0.06^{\ddagger}$ \\
\hline Diastolic blood pressure $(\mathrm{mmHg})$ & $82(12)$ & $-0.10^{\star}$ & -0.03 \\
\hline CHD (\%) & 15.3 & -0.02 & $0.05^{\ddagger}$ \\
\hline CVD (\%) & 17.0 & -0.04 & 0.02 \\
\hline Current smoking (\%) & 33.1 & $0.23^{*}$ & $0.12^{*}$ \\
\hline Diabetes $(\%)$ & 3.3 & $-0.07^{\dagger}$ & -0.01 \\
\hline Vigorous physical activity ( $>3 \mathrm{~h} /$ week) (\%) & 10.9 & 0.01 & -0.01 \\
\hline
\end{tabular}

${ }^{\star} P<0.001 ;{ }^{\dagger} P<0.01 ;{ }^{\ddagger} P<0.05$

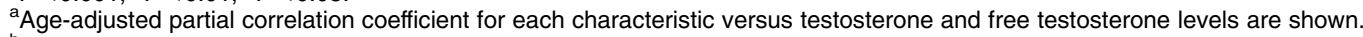

${ }^{\mathrm{b}}$ Baseline characteristic was log transformed for correlation analysis. 
Table 2 Adjusted hazard ratios (HR) of endogenous sex hormones by 1 s.D. increase for all-cause mortality, mortality from cardiovascular disease (CVD), and mortality from ischemic heart disease (IHD). The Tromsø Study 1994-2007.

\begin{tabular}{|c|c|c|c|c|}
\hline & Events $(n)$ & Mean hormone level & Model $1^{\mathrm{a}}$ HR $(95 \% \mathrm{Cl})$ & Model $2^{\mathrm{b}}$ HR $(95 \% \mathrm{Cl})$ \\
\hline All-cause mortality & 395 & & & \\
\hline Total testosterone & & 13.3 & $0.98(0.88-1.09)$ & $0.99(0.89-1.11)$ \\
\hline Free testosterone & & 206 & $0.96(0.85-1.08)$ & $0.94(0.83-1.06)$ \\
\hline Estradiol $^{\mathrm{C}}$ & & 0.06 & $1.04(0.93-1.16)$ & $1.02(0.92-1.14)$ \\
\hline CVD & 133 & & & \\
\hline Total testosterone & & 13.3 & $0.90(0.75-1.07)$ & $0.96(0.80-1.15)$ \\
\hline Free testosterone & & 206 & $1.04(0.84-1.28)$ & $0.99(0.80-1.23)$ \\
\hline Estradiol $^{\mathrm{C}}$ & & 0.06 & $1.12(0.92-1.36)$ & $1.13(0.92-1.38)$ \\
\hline IHD & 80 & & & \\
\hline Total testosterone & & 13.3 & $0.93(0.74-1.17)$ & $1.03(0.82-1.29)$ \\
\hline Free testosterone & & 206 & $1.22(0.95-1.56)$ & $1.16(0.90-1.49)$ \\
\hline Estradiol $^{\mathrm{C}}$ & & 0.062 & $1.06(0.83-1.35)$ & $1.07(0.84-1.38)$ \\
\hline
\end{tabular}

${ }^{\text {aAdjusted for age. }}$

${ }^{\mathrm{b}}$ Adjusted for age, systolic blood pressure, HDL/cholesterol ratio, self-reported diabetes, current smoking and waist/hip ratio.

${ }^{\mathrm{c}}$ Estradiol was transformed to natural logarithm, HR are for 1 s.D. increase in the log.

Adjusted HR of endogenous sex hormones by 1 s.D. increase in hormone level for all-cause, CVD, and IHD mortality are presented in Table 2, and there were no significant associations. The analyses were repeated by quartiles of sex hormones, and HR for all-cause mortality are presented in Table 3. There were no significant differences in HR across quartiles, although there was a tendency towards a larger number of deaths from all causes in the lower quartiles of total and free testosterone. When dichotomized (Table 4), there was a significant increase in all-cause mortality risk for men in the lowest quartile of free testosterone compared with the higher quartiles, HR 1.24 (95\% CI 1.01-1.54), after adjustment for age, smoking, diabetes, HDL/cholesterol ratio, and waist/hip ratio. This finding was only borderline significant, HR 1.25 (95\% CI 0.98-1.57), after exclusion of those who died within the first 2 years of follow-up. All findings regarding mortality were substantially unchanged after exclusion of men younger than 50 years old. As shown in Fig. 1, there were fewer deaths from all-cause mortality with increasing decentiles of free testosterone. Figure 2 shows the multiadjusted HR for all-cause mortality with increasing decentiles of free testosterone with the lowest decentile as the reference category. No threshold effect of free testosterone is apparent from the figure.

\section{Sex hormones and first-ever MI}

During an average 9.1 year follow-up (12 089 person years), 146 of the population sample experienced a firstever MI, an incidence rate of 12.1 per 1000 person years.

Table 5 shows age- and multiadjusted HR for incident cases of first-ever MI by continuous increase per S.D. or quartiles of sex hormones, with the lowest quartile as the reference. For any hormone presented, there was no significant change in risk for MI by 1 s.D. increase in hormone level or by different quartiles of hormone level compared with the lowest quartile. Repeated analyses after exclusion of men younger than 50 years old did not change the results.

Table 3 Adjusted hazard ratios (HR) by quartiles of endogenous sex hormones for all-cause mortality. The Tromsø Study $1994-2007$.

\begin{tabular}{|c|c|c|c|c|}
\hline Hormone & Events $(n)$ & Mean hormone level & Model $1^{\text {a }}$ HR $(95 \% \mathrm{Cl})$ & Model $2^{\mathrm{b}}$ HR $(95 \% \mathrm{Cl})$ \\
\hline \multicolumn{5}{|c|}{ Testosterone $(\mathrm{nmol} / \mathrm{l})$} \\
\hline Q1 $(<9.7)$ & 104 & 8.0 & 1.0 (ref) & 1.0 (ref) \\
\hline Q2 (9.7-12.7) & 108 & 11.3 & $0.99(0.76-1.30)$ & $1.06(0.81-1.40)$ \\
\hline Q3 (12.7-16.0) & 85 & 14.3 & $0.84(0.63-1.12)$ & $0.85(0.64-1.15)$ \\
\hline Q4 $(>16.0)$ & 98 & 19.2 & 1.01 (0.77-1.33) & $1.09(0.81-1.46)$ \\
\hline \multicolumn{5}{|c|}{ Free testosterone $(\mathrm{pmol} / \mathrm{l})$} \\
\hline Q1 $(<158)$ & 143 & 130 & 1.0 (ref) & 1.0 (ref) \\
\hline Q2 (158-197) & 102 & 178 & $0.78(0.61-1.01)$ & $0.80(0.62-1.04)$ \\
\hline Q3 (197-242) & 78 & 219 & $0.72(0.55-0.96)$ & $0.73(0.55-0.97)$ \\
\hline Q4 (>242) & 72 & 285 & $0.97(0.72-1.30)$ & $0.92(0.68-1.24)$ \\
\hline \multicolumn{5}{|l|}{ Estradiol (nmol/l) } \\
\hline Q1 $(<0.04)$ & 86 & 0.03 & 1.0 (ref) & 1.0 (ref) \\
\hline Q2 (0.04-0.06) & 47 & 0.05 & $0.82(0.57-1.17)$ & $0.81(0.56-1.16)$ \\
\hline Q3 $(0.06-0.08)$ & 131 & 0.06 & $1.48(1.12-1.94)$ & $1.32(0.99-1.75)$ \\
\hline Q4 (>0.080) & 116 & 0.09 & $0.98(0.74-1.30)$ & 0.98 (0.73-1.30) \\
\hline
\end{tabular}

${ }^{\mathrm{a}}$ Adjusted for age.

${ }^{\mathrm{b}}$ Adjusted for age, systolic blood pressure, HDL/cholesterol ratio, self-reported diabetes, current smoking, and waist/hip ratio. 
Table 4 Hazard ratios (HR) of low total and free testosterone for cause-specific mortality.

\begin{tabular}{lccc}
\hline & Events $(n)$ & Low testosterone HR $(95 \% \mathrm{Cl})$ & Low free testosterone HR $(95 \% \mathrm{Cl})$ \\
\hline Lowest quartile versus higher $^{\mathrm{a}}$ & & & \\
All-cause & 395 & $1.01(0.80-1.27)$ & $1.24(1.01-1.54)$ \\
CVD & 133 & $1.03(0.70-1.53)$ & $0.84(0.58-1.20)$ \\
IHD & 80 & $1.13(0.68-1.89)$ & $0.85(0.53-1.36)$ \\
\hline
\end{tabular}

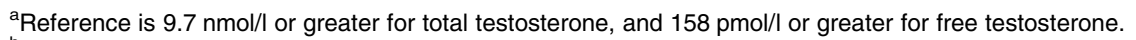

${ }^{\mathrm{b}}$ Adjusted for age, systolic blood pressure, HDL/cholesterol ratio, self-reported diabetes, current smoking, and waist/hip ratio.

\section{Discussion}

In this population-based study, men with free testosterone levels in the lowest quartile were $24 \%$ more likely to die than men with higher levels, a finding that remained significant after multivariate adjustments. We found no significant predictive value of any sex hormone on the risk of death from CVD or IHD, nor on the incidence of first-ever MI. The results were unchanged after exclusion of men younger than 50 years.

Our finding of increased mortality with lower free testosterone is in accordance with recent reports by Laughlin et al. from the Rancho Bernardo study (10) and by Khaw et al. from the EPIC-Norfolk study (11), although their findings were more robust with stronger risk estimates, and with significant associations also with CVD mortality, the latter also including IHD mortality. Maggio et al. (15) reported increased mortality risk when pooling several hormones (testosterone, DHEA, and insulin-like growth factor-1) in the lowest quartile, while Shores et al. (16) reported an increase in mortality risk with lower testosterone levels in a retrospective study of male veterans. As compared with our study, the studies by Laughlin et al. and Khaw et al. had considerably older populations (mean age 71 and 67 years respectively) and a larger proportion of cases. In the population studied by Laughlin et al. with 529 cases compared with our 398, significant increase in risk for all-cause and CVD mortality was only significant when comparing the lowest quartile of total and bioavailable testosterone with the higher quartiles. In the population studied by Khaw et al. with 825 cases, significant reduction in risk for death from all causes and from CVD was found in the third and fourth quartile of total testosterone compared with the lowest, but they found no significant prediction of mortality in stratified analyses of men $<65$ years old. Furthermore, they also predicted IHD mortality only when comparing the lowest with the highest quartile of testosterone. Our study adds evidence about the strength of the impact of free and total testosterone on later risk of morbidity and mortality, in the sense that it contributes to show that large (and rather old) populations with many cases are necessary for differences in later morbidity and mortality across hormone levels to become significant. And furthermore, the influence of testosterone levels may increase with ageing.
While Laughlin et al. demonstrated a tendency towards a threshold effect of lower total testosterone levels on later mortality, this was not apparent in our study.

After exclusion of those who died within the first 2 years of follow-up, the increased risk of death with lower free testosterone levels was only borderline significant in our study, but with a similar risk estimate. One might question that the significant result in the first place is partly due to the contribution from men with subclinical disease at baseline and therefore lower testosterone levels, which died later.

We did not find any significant association between any sex hormone and first-ever MI in the present study. There was, however, a clear tendency towards more MI in the lowest quartile of total and free testosterone. Possibly, the present study with 144 cases of first-ever MI is not sufficiently powered to detect a very slight increase in risk of MI with testosterone levels in the lowest quartile. Nevertheless, previous studies on the prospective relationship between testosterone and MI have also largely reported negative results (4-9). Smith et al. reported increased risk of incident IHD with increased cortisol/testosterone ratio (as an indicator of chronic stress) in the Caerphilly study (17), but they did not examine for the independent contribution of testosterone.

Prospective observational studies have reported increased progression in atherosclerosis $(18,19)$, and although reports from animal studies have been conflicting, in most of them testosterone supplementation led to a decrease in atherosclerotic plaque

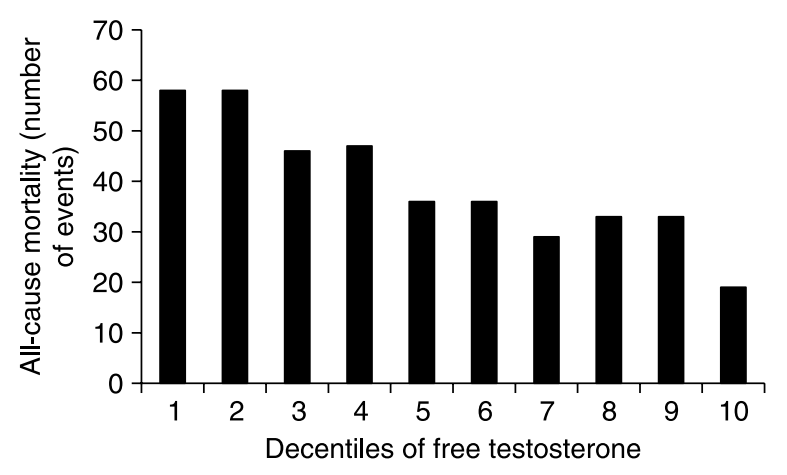

Figure 1 Number of deaths from all causes by decentiles of free testosterone. 


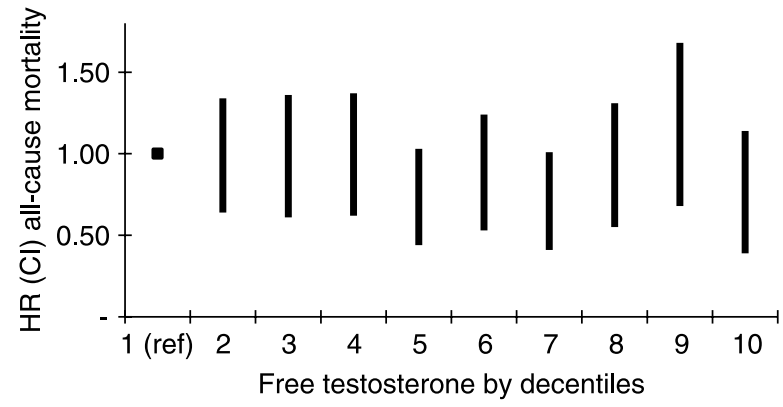

Figure 2 Multiadjusted HR by Cox proportional hazard regression for all-cause mortality by increasing decentile of free testosterone compared with the lowest decentile.

formation (20). Although there are many unanswered questions regarding the mechanism by which testosterone may affect the process of atherosclerosis and risk of death, one proposed mechanism is that testosterone acts through modulation of classical cardiovascular risk factors, supported by findings of a favourable relationship with HDL-cholesterol (21), blood pressure and ventricular mass (22), waist circumference (23), and HbA1c (24). Furthermore, testosterone supplementation of hypogonadal men shifts cytokine balance towards less inflammation (25), which plays a key pathophysiological role in plaque formation. However, associations with testosterone and atherosclerosis after multivariate adjustments for classical risk factors, as is reported, suggest an independent effect of testosterone, or alternatively, confounding by or mediation through factors not measured. The latter is hard to exclude in epidemiological studies, but confounding by or effect mediation through inflammatory markers or endothelial function, for instance, may be possible pathways not accounted for in many observational studies. Effects of testosterone supplementation on plaque formation, demonstrated in animal models, may be mediated through aromatization to $\mathrm{E}_{2}(20)$, but there is also evidence of a short-term vasorelaxing effect of testosterone, probably via non-genomic mechanisms (26). In addition, there are reports from RCTs on improvement in cardiac ischemia in men with coronary disease with short-term testosterone supplementation, as measured by exercise stress testing $(27,28)$. But still, although the literature on the impact of higher endogenous testosterone levels is promising in relation to risk factors, atherosclerosis and short-term beneficial effects on the vasculature, the association between low testosterone and CVD end points do not seem to be very strong, as very large cohorts are needed to find significant associations. One might suggest, in light of the prospective evidence available, that the variations in endogenous testosterone levels in men are not the most important modulator of the development of CVD.

Smoking is strongly associated with mortality and morbidity, and is also positively associated with testosterone levels in most large population-based studies (29). Thus, in any study investigating mortality, smoking is an important confounder, and in our study even more so, due to the effect of smoking on testosterone levels. Smoking has also been associated with higher SHBG levels (29). SHBG is the main carrier of testosterone, and $\sim 65-80 \%$ of the circulating total testosterone is inactive and tightly bound to SHBG. The biologically active fraction circulates either in the free form in the circulation or is loosely bound to albumin. As SHBG levels are reported to be higher in

Table 5 Hazard ratios (HR) by continuous increase per s.D. or quartiles of sex hormones for incident cases of first-ever myocardial infarction in 1318 men. The Tromsø Study 1994-2007.

\begin{tabular}{|c|c|c|c|c|}
\hline Hormone quartiles & Events $(n)$ & Mean hormone level & Model $1^{a}$ HR $(95 \% \mathrm{Cl})$ & Model $2^{b}$ HR $(95 \%$ Cl) \\
\hline \multicolumn{5}{|l|}{ Testosterone (nmol/l) } \\
\hline Whole cohort per 1 s.D. & 144 & 12.8 & $0.94(0.74-1.11)$ & $1.03(0.86-1.23)$ \\
\hline Q1 (<9.7) & 37 & 8.0 & 1.0 (ref) & 1.0 (ref) \\
\hline Q2 (9.7-12.8) & 50 & 11.5 & $1.40(0.91-2.14)$ & $1.50(0.97-2.32)$ \\
\hline Q3 (12.8-16.2) & 25 & 14.4 & $0.72(0.43-1.19)$ & $0.87(0.52-1.46)$ \\
\hline Q4 (>16.2) & 32 & 19.3 & $0.90(0.56-1.44)$ & $1.14(0.68-1.89)$ \\
\hline \multicolumn{5}{|l|}{ Free testosterone $(\mathrm{pmol} / \mathrm{l})$} \\
\hline Whole cohort per 1 s.D. & 143 & 198 & $1.02(0.84-1.25)$ & $1.01(0.83-1.24)$ \\
\hline Q1 (<159) & 48 & 130 & 1.0 (ref) & 1.0 (ref) \\
\hline Q2 (159-198) & 34 & 179 & $0.78(0.50-1.21)$ & $0.76(0.48-1.18)$ \\
\hline Q3 (198-241) & 34 & 219 & $0.94(0.60-1.48)$ & $0.94(0.60-1.48)$ \\
\hline Q4 (>241) & 27 & 287 & $1.02(0.62-1.70)$ & $0.96(0.58-1.59)$ \\
\hline \multicolumn{5}{|l|}{ Estradiol $(\mathrm{nmol} / \mathrm{l})^{\mathrm{c}}$} \\
\hline Whole cohort per 1 S.D. & 138 & 0.06 & $0.961(0.806-1.145)$ & $0.977(0.816-1.169)$ \\
\hline Q1 $(<0.04)$ & 35 & 0.03 & $1.0(\mathrm{ref})$ & 1.0 (ref) \\
\hline Q2 (0.04-0.06) & 19 & 0.05 & $1.01(0.58-1.77)$ & $0.99(0.56-1.74)$ \\
\hline Q3 $(0.06-0.08)$ & 46 & 0.06 & $1.33(0.85-2.07)$ & $1.23(0.78-1.93)$ \\
\hline Q4 (>0.08) & 38 & 0.09 & $0.96(0.60-1.53)$ & $0.98(0.61-1.55)$ \\
\hline
\end{tabular}

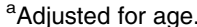

${ }^{\mathrm{b}}$ Adjusted for age, systolic blood pressure, HDL/cholesterol ratio, self-reported diabetes, current smoking, and waist/hip ratio.

${ }^{c}$ When used as a continuous variable, estradiol was transformed to natural logarithm; HR are for 1 s.D. increase in the log. 
smokers while bioavailable testosterone levels have been unaffected by smoking, English et al. (30) suggested in a case-control study of 50 men that the increase in total testosterone levels may have been secondary to the raised SHBG levels. However, smoking has also been shown to increase free testosterone, which should not be affected in the same way by SHBG levels. Furthermore, both total and free testosterone increases gradually with increasing number of cigarettes smoked daily, and could therefore possibly mask borderline hypogonadism. However, adjusting or not adjusting for smoking did not affect the outcome in the present study.

Besides being a large population-based study, the main strength with our study is the thorough ascertainment of end points, which is possible due to our national registry on mortality. However, there are also limitations to our study, of which several are partly due to the fact that the Tromsø Study was not set up to study effects of testosterone levels on mortality and morbidity. Blood samples were drawn between 0800 and $1600 \mathrm{~h}$. They should preferably have been drawn in the morning, as there is a certain diurnal variation in testosterone levels. However, the diurnal variation is less pronounced as men age (31), and our population had a mean age of 59 years. Blood samples were frozen for 7 years before they first were thawed for analysis in 2001. When stored frozen, levels of steroid hormones have been shown to be relatively stable for a period up to 10 years $(32,33)$. Furthermore, a slight decrease in hormone levels would not be expected to alter the ordinal associations for the observed levels. The day-to-day variation in testosterone levels may be considerable, for which reason repeated tests are recommended for a diagnosis of hypogonadism. With one single blood sample, we did not account for the intraindividual day-to-day variation in our study. Nevertheless, those are all sources of potential nondifferential misclassification, tending to attenuate possible associations rather than to produce spurious effects of sex hormones on later mortality. And finally, as always in epidemiological studies, residual confounding by unknown factors can never be fully accounted for.

In conclusion, men with the lowest free testosterone levels had a $24 \%$ increased risk of death from all-cause mortality. In view of our sample size and other limitations, our findings are fairly consistent with previous prospective studies to date, and should further encourage larger testosterone supplementation trials for the definitive assessment of long-term health benefits.

\section{Declaration of interest}

The authors declare that there is no conflict of interest that could be perceived as prejudicing the impartiality of the research reported.

\section{Funding}

This work was supported by a grant from The Northern Norway Region Health Authority.

\section{References}

1 Gray A, Feldman HA, McKinlay JB \& Longcope C. Age, disease, and changing sex hormone levels in middle-aged men: results of the Massachusetts male aging study. Journal of Clinical Endocrinology and Metabolism 199173 1016-1025.

2 Barrett-Connor E \& Bhasin S. Time for (more research on) testosterone. Journal of Clinical Endocrinology and Metabolism 2004 89 501-502.

3 Liu PY, Death AK \& Handelsman DJ. Androgens and cardiovascular disease. Endocrine Reviews 200324 313-340.

4 Yarnell JW, Beswick AD, Sweetnam PM \& Riad-Fahmy D. Endogenous sex hormones and ischemic heart disease in men. The Caerphilly prospective study. Arteriosclerosis and Thrombosis 199313 517-520.

5 Phillips GB, Yano K \& Stemmermann GN. Serum sex hormone levels and myocardial infarction in the Honolulu Heart Program. Pitfalls in prospective studies on sex hormones. Journal of Clinical Epidemiology 198841 1151-1156.

6 Contoreggi CS, Blackman MR, Andres R, Muller DC, Lakatta EG, Fleg JL \& Harman SM. Plasma levels of estradiol, testosterone, and DHEAS do not predict risk of coronary artery disease in men. Journal of Andrology 199011 460-470.

7 Mikulec KH, Holloway L, Krasnow RE, Javitz H, Swan GE, Reed T, Marcus R \& Carmelli D. Relationship of endogenous sex hormones to coronary heart disease: a twin study. Journal of Clinical Endocrinology and Metabolism 200489 1240-1245.

8 Hautanen A, Mänttäri M, Manninen V, Tenkanen L, Huttunen JK, Frick MH \& Adlercreutz H. Adrenal androgens and testosterone as coronary risk factors in the Helsinki Heart Study. Atherosclerosis 1994105 191-200.

9 Cauley JA, Gutai JP, Kuller LH \& Dai WS. Usefulness of sex steroid hormone levels in predicting coronary artery disease in men. American Journal of Cardiology 198760 771-777.

10 Laughlin GA, Barrett-Connor E \& Bergstrom J. Low serum testosterone and mortality in older men. Journal of Clinical Endocrinology and Metabolism 200893 68-75.

11 Khaw KT, Dowsett M, Folkerd E, Bingham S, Wareham N, Luben R, Welch A \& Day N. Endogenous testosterone and mortality due to all causes, cardiovascular disease, and cancer in men: European prospective investigation into cancer in Norfolk (EPIC-Norfolk) prospective population study. Circulation 2007116 2694-2701.

12 Svartberg J, Midtby M, Bønaa KH, Sundsfjord J, Joakimsen RM \& Jorde R. The associations of age, lifestyle factors and chronic disease with testosterone in men. The Tromsø Study. European Journal Endocrinology 2003149 145-152.

13 Vermeulen A, Verdonck L \& Kaufman JM. A critical evaluation of simple methods for the estimation of free testosterone in serum. Journal of Clinical Endocrinology and Metabolism $1999 \mathbf{8 4}$ 3666-3672.

14 Yusuf S, Hawken S, Ounpuu S, Dans T, Avezum A, Lanas F, McQueen M, Budaj A, Pais P, Varigos J \& Lisheng L. Effect of potentially modifiable risk factors associated with myocardial infarction in 52 countries (the INTERHEART study): case-control study. Lancet $2004 \mathbf{3 6 4}$ 937-952.

15 Maggio M, Lauretani F, Ceda GP, Bandinelli S, Ling SM, Metter EJ, Artoni A, Carassale L, Cazzato A, Ceresini G, Guralnik JM, Basaria S, Valenti G \& Ferrucci L. Relationship between low levels of anabolic hormones and 6-year mortality in older men. The aging in the Chianti Area (InCHIANTI) study. Archives of Internal Medicine $20071672249-2254$.

16 Shores MM, Matsumoto AM, Sloan KL \& Kivlahan DR. Low serum testosterone and mortality in male veterans. Archives of Internal Medicine 2006166 1660-1665.

17 Smith GD, Ben-Shlomo Y, Beswick A, Yarnell J, Lightman S \& Elwood P. Cortisol, testosterone, and coronary heart disease: prospective evidence from the Caerphilly study. Circulation 2005 $112332-340$. 
18 Hak AE, Witteman JC, de Jong FH, Geerlings MI, Hofman A \& Pols HA. Low levels of endogenous androgens increase the risk of atherosclerosis in elderly men: the Rotterdam study. Journal of Clinical Endocrinology and Metabolism 200287 3632-3639.

19 Muller M, van den Beld AW, Bots ML, Grobbee DE, Lamberts SW \& van der Schouw YT. Endogenous sex hormones and progression of carotid atherosclerosis in elderly men. Circulation 2004109 2074-2079.

20 McGrath KC, McRobb LS \& Heather AK. Androgen therapy and atherosclerotic cardiovascular disease. Vascular Health and Risk Management 20084 11-21.

21 Haffner SM, Mykkänen L, Valdez RA \& Katz MS. Relationship of sex hormones to lipids and lipoproteins in nondiabetic men. Journal of Clinical Endocrinology and Metabolism $1993 \quad \mathbf{7 7}$ $1610-1615$.

22 Svartberg J, von Mühlen D, Schirmer H, Barrett-Connor E, Sundsfjord J \& Jorde R. Association of endogenous testosterone with blood pressure and left ventricular mass in men. The Tromsø Study. European Journal Endocrinology 2004150 65-71.

23 Svartberg J, von Mühlen D, Sundsfjord J \& Jorde R. Waist circumference and testosterone levels in community dwelling men. The Tromsø Study. European Journal of Epidemiology 200419 657-663.

24 Svartberg J, Jenssen T, Sundsfjord J \& Jorde R. The associations of endogenous testosterone and sex hormone-binding globulin with glycosylated hemoglobin levels, in community dwelling men. The Tromsø Study. Diabetes \& Metabolism 200430 29-34.

25 Malkin CJ, Pugh PJ, Jones RD, Kapoor D, Channer KS \& Jones TH. The effect of testosterone replacement on endogenous inflammatory cytokines and lipid profiles in hypogonadal men. Journal of Clinical Endocrinology and Metabolism 200489 3313-3318.
26 Yildiz O \& Seyrek M. Vasodilating mechanisms of testosterone. Experimental and Clinical Endocrinology and Diabetes 2007115 1-6.

27 Jaffe MD. Effect of testosterone cypionate on postexercise ST segment depression. British Heart Journal 197739 1217-1222.

28 English KM, Steeds RP, Jones TH, Diver MJ \& Channer KS. Lowdose transdermal testosterone therapy improves angina threshold in men with chronic stable angina: a randomized, double-blind, placebo-controlled study. Circulation 2000102 1906-1911.

29 Svartberg J \& Jorde R. Endogenous testosterone levels and smoking in men. The fifth Tromsø Study. International Journal of Andrology 200730 137-143.

30 English KM, Pugh PJ, Parry H, Scutt NE, Channer KS \& Jones TH. Effect of cigarette smoking on levels of bioavailable testosterone in healthy men. Clinical Science 2001100 661-665.

31 Bremner WJ, Vitiello MV \& Prinz PN. Loss of circadian rhythmicity in blood testosterone levels with aging in normal men. Journal of Clinical Endocrinology and Metabolism 198356 1278-1281.

32 Kley HK, Schlaghecke R \& Krüskemper HL. Stability of steroids in plasma over a 10-year period. Journal of Clinical Chemistry and Clinical Biochemistry 198523 875-878.

33 Bolelli G, Muti P, Micheli A, Sciajno R, Franceschetti F, Krogh V, Pisani P \& Berrino F. Validity for epidemiological studies of longterm cryoconservation of steroid and protein hormones in serum and plasma. Cancer Epidemiology, Biomarkers \& Prevention 19954 509-513.

Received 6 June 2009

Accepted 16 June 2009 